Instituto Internacional de Investigación y Desarrollo Tecnológico Educativo INDTEC, C.A. DOI: https://doi.org/10.29394/Scientific.issn.2542-2987.2019.4.13.19.346-362

OAl-PMH: $\underline{\text { http://www.indteca.com/ojs/index.php/Revista Scientific/oai }}$

Ensayo Original / Original Essay

\title{
Epistemología del Pensamiento Pedagógico como Eje Transformador en la Praxis Educativa
}

Autoras: Marianny del Carmen Acosta Vega Universidad Nacional Experimental "Rafael María Baralt", UNERMB mariannyacosta3@gmail.com Zulia, Venezuela https://orcid.org/0000-0001-9813-8304

Maibelyn del Carmen Acosta Vega Universidad Nacional Experimental "Rafael María Baralt", UNERMB maibelyn19@gmail.com Zulia, Venezuela https://orcid.org/0000-0001-8492-657X

\section{Resumen}

El propósito del presente ensayo es destacar las bases epistemológicas en la formación pedagógica del docente en Venezuela, para así incentivar a la formación docente crítica y reflexiva, bajo fundamentos filosóficos y pedagógicos, tales como el Ideario Bolivariano de Simón Rodríguez (17691854); el pedagogo Luis Beltrán Prieto Figueroa (1902-1993); la educadora Belén San Juan (1916-2004) y de esta manera lograr una verdadera transformación en la praxis educativa. Para ello se realizó una investigación de carácter documental, siendo la revisión bibliográfica la técnica de recolección de información. Finalmente se esboza la visualización de docentes centrados en la investigación y en la práctica reflexiva de la acción.

Palabras clave: epistemología; pedagogía; educación.

Fecha de Recepción: 06-11-2018
Fecha de Aceptación: 16-03-2019
Fecha de Publicación: 05-08-2019 


\title{
Epistemology of Pedagogical Thinking as a Transforming Axis in Educational Praxis
}

\begin{abstract}
The purpose of this essay is to highlight the epistemological bases in teacher training in Venezuela, in order to encourage critical and reflective teacher training, under philosophical and pedagogical foundations, such as the Bolivarian Ideology of Simón Rodríguez (1769-1854); the pedagogue Luis Beltrán Prieto Figueroa (1902-1993); the educator Belén San Juan (19162004) and in this way achieve a true transformation in educational praxis. To this end, a documentary research was carried out, with the bibliographic review being the technique for collecting information. Finally, the visualization of teachers focused on research and reflective practice of action is outlined.
\end{abstract}

Keywords: epistemology; pedagogy; education.

Date Received: 06-11-2018
Date Acceptance:

16-03-2019
Date Publication:

05-08-2019 


\section{Introducción}

El aprendizaje en todo ser humano es constante, todos los días se aprende algo nuevo, el deseo de saber y conocer se ha valorado en el individuo a lo largo del tiempo. En el hombre siempre ha existido la curiosidad por lo que ocurre en el mundo, todo esto ha impulsado a buscar razones y causas, a descubrir el por qué y el cómo, lo que conlleva así a poder determinar las consecuencias de las cosas, de esta forma siempre se mantiene una inquietud constante sobre el sentido que tiene su vida. Mediante estas indagaciones el hombre logra adquirir nuevos conocimientos que dan respuestas que le otorgan satisfacción, pero a su vez más dudas y las ansias de indagar crecen hasta que llega a comprender la esencia de la vida, el poder que tiene la mente y todos esos fenómenos inexplicables.

Extrapolando las ideas al ámbito de la educación, esta tiene como función principal el mejoramiento continuo de los individuos y de las sociedades, no solo ofrece un beneficio personal o proveer al mundo económico de personal calificadas, la finalidad de la educación radica en facilitar al educando la adquisición de conocimientos y sobre todo valores que le permitan desarrollarse de manera favorable por medio de la realización de aptitudes y talentos de cada individuo. Es importante destacar que la educación actual está atravesando un proceso de cambio en su identidad para poder transformarse en una estructura más flexible lo cual posibilite un camino social al discernimiento y así lograr un avance en los individuos con base a las necesidades que se demanda en este siglo.

En el caso específico de Venezuela, la educación ha evolucionado paulatinamente, por ende se han planteado y desarrollado políticas educativas que dan respuestas a las necesidades que no han sido cubiertas en la mayoría de los casos, las cuales garantizan que la educación pueda llegar a todos los estratos sociales y de esta forma a todos los niveles del sistema, esto se logra mediante los ordenamientos jurídicos contemplados en la Constitución de la 
República Bolivariana de Venezuela (1999), aún vigente y el ente rector y orientador como es el Ministerio del Poder Popular para la educación, como se contempla en el artículo 102 de la Constitución: "La educación es un derecho humano y un deber social fundamental, es democrática, gratuita y obligatoria... La educación es un servicio público y está fundamentada en el respeto a todas las corrientes del pensamiento..." (págs. 20-21).

Bajo estos preceptos, se puede establecer que el nuevo currículo sea de eje central, de esta manera la formación del docente de aula sea más flexible, integral, y que este en una constante construcción a fin de garantizar el desarrollo pedagógico pertinente, en concordancia con el Proyecto Educativo Nacional, establecido en el año 2001, para así lograr una coherencia entre la teoría y las prácticas educativas.

Actualmente, el docente debe ser visualizado como un mediador, un investigador constante, debe ser innovador, empático. A tenor de lo expuesto, se aspiran percibir las exigencias derivadas de la sociedad y visualizar el país que se quiere construir, internalizando el porqué de un currículo global, flexible, continuo, transversal y transdisciplinario, en el cual es imperante que los docentes se centren en investigar y en tener una práctica critico reflexiva en la acción. Por lo tanto, se debe tener en cuenta todos los modelos del pensamiento pedagógico con el propósito de generar un mayor potencial en cada persona tomando en cuenta la sociedad donde se desenvuelve, su etnia y su cultura.

Ahora bien, vale la pena preguntarse si existe una verdadera adaptación entre las propuestas educativas trazadas en el currículo y lo que verdaderamente ocurre en la praxis educativa venezolana, por ende, sería bueno plantearse, cual ha sido el desarrollo de nuestro sistema educativo ya que los cambios nominales son de gran importancia para poder entender el presente y de esta forma comprender el futuro de la educación en nuestro país.

Recordando que, cuando se habla de epistemología de la educación, 
esta se debe considerar como un espacio que sirve para analizar la situación de una manera crítica reflexiva, para así lograr una diagnosis tanto de los logros como de las dificultades que se presentan, y de esta manera, enfrentar temas fundamentales en la generación de pensamiento pedagógico. Por su parte, Moreno (2018), plantea que "La esencia de la epistemología radica en el tratado del conocimiento general, la ciencia, los problemas de ser abordado en un contexto" (pág. 366).

Epistemológicamente, la investigación no se limita sólo a conocer teorías educativas de diversos autores, sino a comprender cuáles son las condiciones y procedimientos que hacen posible que un autor o autora realice una propuesta educativa, lo que conlleva a plantearse que actualmente en la educación venezolana existe una ruptura epistémica del currículo actual. Es posible que este análisis nos ayude pensar y construir la educación desde una perspectiva diferente y facilite elaborar nuestras propias teorías educativas de una forma reflexiva y colaborativa, transformándonos así, en autores de nuestro propio saber.

Frente a este escenario, el objetivo de dicho ensayo es destacar las bases epistemológicas en la formación pedagógica del docente en Venezuela, para así incentivar a la formación docente crítica y reflexiva, bajo fundamentos filosóficos y pedagógicos, y de esta manera lograr una verdadera transformación en la praxis educativa.

\section{Desarrollo}

La educación es un proceso bastante complejo al igual que el modelo educativo venezolano, por ende, siempre debe existir la constate reflexión de cómo se puede fortalecer dicho proceso, quizás en materia educativa es bueno plantearse las contradicciones que existe entre la praxis y la teoría y de esta manera vislumbrar las diferencias que existente entre el discurso y la acción en el hecho educativo, debido a que muchas veces las preguntas son más 
esenciales que las respuestas.

Conviene destacar, que para el logro de un cambio significativo en cuanto a la educación es imperante estudiar el pensamiento interdisciplinario, y este se ha encontrado principalmente relacionado con el ámbito psicopedagógico, diferentes autores contemporáneos han desarrollo teorías, entre ellos, Contreras José (1985); Gimeno José (1987); Goodman Jesse (1987); Zeicher Kenneth (1995); Canfux Verónica (2000), dichos autores consideran que el pensamiento pedagógico se refiere al pensamiento del profesor en relación con su tarea docente educativa, lo cual ocurre al valorar esta tarea como la actividad intelectual que exige dicha actividad desde el enfoque científico. Por esta razón, Gómez y Pulido (2016), considera que:

...el maestro y el pedagogo ya no pertenecen a una tradición o a un modelo pedagógico, o a una tendencia que del pasado le aporta andamiajes teóricos que determinan sus actividades y acciones, sino que el pertenecer al ahora, al presente, le permite relacionarse con un conjunto amplio de situaciones, contextos, formas de vida, formas y prácticas de institucionalización y maneras de sentir y hacer pedagogía o pedagogías, pues se convierte en el objeto de su reflexión, es decir, la formación ubicada en "las entrañas del presente"; el pedagogo y el maestro no pueden dejar de lado su cuestionamiento y su pertenencia al "nosotros", es decir, al conjunto de relaciones culturales propias de su actualidad (pág. 11).

De esta manera, al estudiar el término pedagogía en el ámbito de la educación como ciencia, se puede establecer que la pedagogía no se trata solo de una alocución sobre la educación, sino más bien sobre la práctica que tiene cada docente en el aula de clases, para de esta formar facilitar la construcción del conocimiento con todo lo que esto conlleva. Asimismo, la educación es transformadora de sujetos, quienes a su vez transforman la práctica social.

Ahora bien, en el caso específico de Venezuela en año 1999, surge lo 
que se conoce como educación Bolivariana, como nuevo paradigma emergente, junto al Proyecto Educativo Nacional, en el cual se plantea un modelo pedagógico de equilibrio social el cual facilita el cumplimiento de los criterios constitucionales para así garantizar una educación integral y de calidad, lo que conlleva alcanzar una formación completa que permita cumplir con lo exigido en la sociedad actual, como lo menciona Figueroa (2014):

...El Estado debe estar dispuesto a asumir con responsabilidad técnica profesional y compromiso revolucionario el mandato social de monitorear las políticas públicas en general y las educativas en particular y con ello de ejecutar labores de seguimiento, supervisión, acompañamiento pedagógico y evaluación de la calidad de la educación que ya no pueden estar enmarcadas en el simple ejercicio profesional punitivo sino más bien dignificadas desde el ejercicio de la autoridad moral de quienes asuman esta tarea (pág. 260).

Por lo tanto la educación venezolana debe responder a las exigencias de esta concepción curricular la cual está orientada principalmente bajo fundamentos filosóficos del Sistema Educativo Bolivariano, plasmado en el ideario Bolivariano, conocido como el árbol de las tres raíces, creado en los años 80, por un movimiento secreto revolucionario, el cual recoge los pensamientos de nuestro Libertador Simón Bolívar (1783-1830), el gran educador Simón Rodríguez (1769-1854) y Ezequiel Zamora (1817-1860) conocido como el general del pueblo. Epistemológicamente, para ellos la refundación de la república se basaba en principios de igualdad, libertad y justicia, logrando así una ruptura al paradigma imperante en la época colonialista.

El árbol de las tres raíces, es un gran legado que nos permite comprender el pasado y vislumbrar el futuro de la educación venezolana, se puede afirmar que las ideas rectoras del gran maestro Simón Rodríguez es la fuente filosófica más importante de la educación Bolivariana, conocida actualmente como Pensamiento Robinsoniano, dicho pensamiento parte 
principalmente de la concepción que la educación debe ser popular y social, y que debía existir una estrecha relación entre la educación y el trabajo, promoviendo así la creación de escuelas técnicas.

Cabrera, Pérez y González (2012), basados en las concepciones pedagógicas imperantes en el Currículo Nacional Bolivariano sobre del modelo de Simón Rodríguez, resumen que:

...Se debe ir haciendo del perfil del maestro, la síntesis de todas las virtudes humanas, que sin ser un sabio, debe ser una persona: distinguida por su educación, por la pureza de sus costumbres, jovial, accesible, dócil, franca, en fin en quien se encuentre mucho que imitar y poco que corregir (pág. 400).

Asimismo, para Simón Rodríguez la escuela no era solo un medio para enseñar, este concebía la educación como una vía que le permitiera a cada ser humano el desarrollo a plenitud de sus talentos, para así lograr formarse como persona y como ciudadano solidario y productivo, con sentido para la vida, amor a libertad, asegurando así la verdadera independencia de las Américas, sin copias de otras sociedades.

En cuanto a los fundamentos pedagógicos que se destacan en el Sistema Educativo Venezolano, se encuentran las ideas del ilustre pedagogo Luis Beltrán Prieto Figueroa (1902-1993), promotor del Estado Docente, la Nueva Escuela y el Humanismo Democrático, era partidario de una educación orientada a elevar el nivel de vida de toda la población no de una parte de ella, en tal sentido, debía ser democrática, humana, gratuita y obligatoria, en concordancia a la igualdad de oportunidades en base a las capacidades de cada ser humano.

Sobre estos ideales, se estableció una estrategia educativa a nivel nacional, el cual se conoce actualmente como Estado docente, en el cual aprueba la intervención del estado en la educación, ya que como institución pública y social, tiene la autoridad de establecer leyes que regulen la sociedad, 
velando así por las necesidades del pueblo.

Figueroa (1990a), afirma en su obra El Estado y la Educación en América Latina, que:

El Estado interviene, por derecho propio, en la organización de la educación del país, y orienta, según su doctrina política, esa educación. Depende la orientación de una escuela de la orientación política del Estado. Si el Estado es fascista, la escuela es fascista. Si el Estado es nazista, la escuela es nazista. Si el Estado es falangista, la escuela es falangista. $Y$ si el Estado es democrático, la orientación de la escuela necesariamente tiene que ser democrática. En efecto, en toda sociedad la educación sirve a elevados fines sociales, pero no le corresponde fijar autónomamente sus propias metas. Obedece su orientación a la sociedad donde actúa (pág. 27).

Otro de sus aportes a la educación fue el Humanismo democrático, en el cual coincide con los ideales del brasileño Paulo Freire (1921-1997), la sociedad requiere de ciudadanos que sean capaces de contribuir al desarrollo del país, no de manera individualizada, sino para el beneficio del colectivo, con esta teoría trata de perfeccionar las cualidades del hombre fortaleciendo sus valores, así Figueroa (1990b), enfatiza:

En una democracia ordenada y planificada la formación educativa de los ciudadanos se realiza atendiendo a los requerimientos que el desarrollo económico y social demanda (...) son los hombres formados los que generan desarrollo y riqueza (...) aparejado a la redistribución del ingreso, tal como acontece en la democracia socialista (...) la educación democrática es gratuita y obligatoria; tiende a dar a los ciudadanos igualdad en las oportunidades para alcanzar todos los grados y ventajas que corresponden a los ciudadanos en democracia (pág. 55-59).

En efecto, Luis Beltrán Prieto Figueroa, es uno de los personajes más destacados en la formación del sistema educativo venezolano, sus ideales en cuanto a la educación se puede resumir, en una educación democrática, con igualdad y sin exclusiones, fundamentado en las raíces de nuestra identidad, 
desde una perspectiva humanista considerando las diversas etnias y culturas, donde cada persona tenga las mismas oportunidades, formando individuos integrales, libres, responsables, en aras del bienestar tanto personal como de la sociedad.

Otro de los aportes para la formación pedagógica en el sistema educativo venezolano, fueron las ideas que implanto en escuelas experimentales la educadora Belén San Juan (1916-2004), firme seguidora de los postulados de Simón Rodríguez y que junto a Luis Beltrán Prieto Figueroa, fundó en el año 1955 el instituto de educación integral, conocido como el laboratorio pedagógico, el cual uso para profundizar en sus pensamientos, todo esto sirvió como pilar para el nuevo currículo.

Belén San Juan, asume la educación en las aulas de clases de manera integral, es decir preparando para la vida, incluye ideas de formación para la paz y que la enseñanza se da a través de la experiencia, mediante métodos pedagógicos que incluyen todos los saberes, formando además en ellos un profundo sentimiento por la patria.

Una vez esbozados las bases epistemológicas de la formación pedagógica presentes en el currículo actual del sistema educativo venezolano, es oportuno preguntarse: ¿Existe una concordancia entre lo planteado en el nuevo currículo y la realidad en la praxis educativa?. Ante esta interrogante, se puede afirmar que existe una ruptura epistémica entre el pensamiento pedagógico imperante y la praxis educativa.

Por su parte, Matos, Cegarra y Rivera (2017): consideran como praxis educativa aquella que "lleva al docente a tomar conciencia de la necesidad de formarse para transformar su accionar educativo, desde esa relación dialógica de saberes y conocimientos para promover un proceso interactivo afectivo, cognitivo y práctico entre sus compañeros y estudiantados" (pág. 331); el cumplimiento de esto conlleva a una verdadera transformación de la educación. 
Ahora bien, a pesar del tiempo de aplicación de este nuevo paradigma en las escuelas, los docentes no están preparados para este nuevo cambio, para ello se debe tener una concepción holística, actuar bajo posturas paradigmáticas del saber, por lo tanto su visión epistemológica debe ser distinta a la de la educación tradicional en la cual son visto como dadores de clases, es decir solo imparten conocimiento, los docentes deben ser visto realmente como educadores, donde los estudiantes tengan una participación activa en el aula de clases.

Al respecto, Obando (2019), realizó una investigación titulada, El acompañamiento pedagógico y la praxis educativa en la educación básica en Venezuela, en la cual afirma que "...se sigue realizando una praxis educativa dogmática, para formatear el cerebro de los estudiantes, con la información requerida para domesticar y amansar a los seres humanos en función de la preservación de sociedades de la explotación y dominación" (pág. 98); esto indica que subsiste la tendencia a resistir el cambio, aplicando viejos paradigmas.

Razón por la cual, se puede afirmar que todos estos nuevos proyectos y propuestas educativas en teoría están muy de acorde con el deber ser, pero la praxis se ha encargado de convertirlo en una utopía, esto se debe a que el docente no termina de internalizar los lineamentos, adicionando a esto que en el hecho educativo el discurso se distorsiona con la divulgación de valores partidistas convirtiendo las aulas de clases en reuniones políticas. Es importante destacar que la docencia no puede ejercerse coactivamente, ni por tendencias políticas ni mucho menos con fundamento a una remuneración, ya que esto llevaría a un camino sin salida, cada docente debe comprender que la docencia es un arte, que ser docente conlleva a más que títulos y credenciales.

Aunando a lo planteado anteriormente, en el Documento La Transformación del Sistema Educativo Venezolano de la Cámara Venezolana 
de Educación Privada (CAVEP, 2014), expresa que:

No basta con tener al alcance el mejor currículo del mundo, es necesario que el docente sepa cómo utilizarlo en el marco contextual de la realidad en la cual se desarrolla el proceso de enseñanza y aprendizaje, de allí que la formación de los docentes, la evaluación constructiva y oportuna del aprendizaje y el perfeccionamiento de los actos de acompañamiento a las aulas, son prioritarios para lograr lo que entendemos como calidad de la educación (pág. 10).

Por otro lado, en todas las instituciones educativas debe existir lo que se conoce como trabajo en equipo, el aula de la clases es el mejor escenario para trabajar en equipo, entendiendo que no basta solo con estar reunidos en un mismo espacio, trabajar en equipo va más allá, es compartir la forma de trabajar y los interés de todas las personas que lo integran, ya que se debe recordar que el aprendizaje es social, lamentablemente en la actualidad de las escuelas se observa carencia de trabajo en equipo, no existe unión entre los participante, los beneficios personales prevalecen sobre beneficios en conjuntos de la institución educativa, lo que genera fallas en la gerencia educativa debido a que cada persona tiene diferentes intereses y por ende diferentes concepciones paradigmáticas.

De igual manera, el docente no puede resignarse al saber parcelado, cada docente debe inspirarse en la búsqueda de un pensamiento multidimensional, sin eliminar la contradicción interior, tomando en cuenta la diversidad, las verdades más profundas que quizás difieran una de otras pero que no dejan de estar relacionadas entre ellas, para lograr una verdadera critica reflexiva de las situaciones que se presenten.

Por último, los docentes deben mostrar empatía hacia sus estudiantes, esta es una de las bases fundamentales para lograr un buen desarrollo académico en cada uno de ellos y lograr así una formación de calidad. Un docente empático crea un ambiente positivo en las aulas de clases, usando 
nuevas estrategias, orientando a sus alumnos de la mejor formar, donde se visualice realmente el desempeño de cada uno, desarrollándose así, como un verdadero educador.

\section{Conclusiones}

Actualmente en la educación venezolana enfrenta un gran desafío, lograr un cambio en la praxis educativa, usando como referentes esos grandes pedagogos venezolanos, que formaron el nuevo currículo por el cual se rige el sistema educativo venezolano. Los docentes deben asumir estas premisas de manera objetiva y critica debido a que este nuevo paradigma educativo emergente implica un cambio de $360^{\circ}$, es decir un estilo de pensamiento y de vida completamente distinto al ejecutado hasta el momento. En consecuencia, el docente debe responder a la misión, visión de la escuela y currículo, de esta manera el docente debe estar siempre listo a aprender, por convicción, motivación propia, por su amor a la sabiduría.

Para lograr una verdadera concordancia entre lo que dice la teoría y lo que se ve en la praxis educativa, debe partirse por lo micro, la transformación de las aulas de clase con docente de verdadera vocación para así lograr un verdadero aprendizaje significativo, en el cual exista la participación activa de los estudiantes, en donde realmente se internalice el conocimiento, y de esta forma preparar al individuo para la vida, de una manera integral, humana, tal como lo platean Luis Beltrán Prieto Figueroa y Belén San Juan, como seguimiento a las ideas del gran educador Simón Rodríguez.

De igual manera, las instituciones educativas deben ser concebidas como organizaciones para la formación de talento humano necesario para la sociedad, debe funcionar en su gerencia tal como lo hacen las empresas de clase mundial para el reclutamiento y selección del personal docente, aplicar el currículo formador de ciudadanos con diferentes características exigidas por la sociedad. 


\section{Reflexiones finales}

A modo de reflexión y como consejo al iniciar la búsqueda de información, no hay que dejarse impresionar por lo impreso en textos o las posturas de doctrinarios o líderes reconocidos, pues quizás en ellos la capacidad de aprender ha caducado. La verdad absoluta no existe, es tarea de cada docente, un constante preguntar e indagar con ansias de saber.

Estudiar no es una competencia cuyo premio es un título académico, debemos buscar el aprendizaje para crecer intelectual y espiritualmente, con la intención de aplicar lo adquirido y seguir a la expectativa de nuevos conocimientos. Razón por la cual, se está obligado a transformarnos realmente, para formarnos como seres humanos pensantes, lo cual nos permita construir la realidad como un tejido multidimensional, con claridad, para poder emitir de manera libre un juicio de ideas y cambiarlas en cualquier momento, sin restricción alguna, pues el ser es capaz de evaluarse constantemente y auto corregirse sin temor, con honestidad y sentimiento de hacer lo correcto.

\section{Referencias}

Cabrera, A., Pérez, Y., \& González, O. (2012), Modelo pedagógico de Simón Rodríguez en el contexto actual venezolano. Impacto Científico, 7(2), 393-401, e-ISSN: 1836-5042. Recuperado de:

http://produccioncientificaluz.org/index.php/impacto/article/view/470

CAVEP (2014). La Transformación del Sistema Educativo Venezolano. Un reto ineludible. Caracas, Venezuela: Documento de la Cámara Venezolana de Educación Privada. Recuperado de:

http://www.cerpe.org.ve/tl files/Cerpe/contenido/documentos/Calidad \%20Educativa/Propuesta\%20Cavep\%20al\%20ME\%20(2).pdf

Constitución de la República Bolivariana de Venezuela (1999). Capítulo VI, de Ios Derechos Culturales y educativos. Gaceta Oficial Extraordinaria 
$N^{\circ} 36.860$ de fecha 30 de diciembre. Caracas, Venezuela: Asamblea Nacional Constituyente. Recuperado de:

https://www.oas.org/dil/esp/constitucion_venezuela.pdf

Figueroa, M. (2014). BONILLA-MOLINA, L. (2014). Calidad de la educación: ideas para seguir transformando la educación. Integración y Conocimiento, N. ${ }^{\circ}$. Venezuela: Ediciones MPPEU-CIM. Recuperado de: https://revistas.unc.edu.ar/index.php/integracionyconocimiento/article/v iewFile/9389/10138

Gómez, L., \& Pulido, O. (2016). La pedagogía y su presente: umbrales y relaciones. Praxis \& Saber, 7(13), 9-14, ISSN: 2216-0159; e-ISSN: 2462-8603. Recuperado de:

http://www.scielo.org.co/pdf/prasa/v7n13/v7n13a01.pdf

Matos, Y., Cegarra, O., \& Rivera, C. (2017). La Praxis Docente desde la Formación Permanente. Revista Scientific, 2(4), 319-336, e-ISSN: 2542-2987. Recuperado de:

https://doi.org/10.29394/scientific.issn.2542-2987.2017.2.4.18.319-336

Moreno, Y. (2018). Epistemología y Pedagogía... Consideraciones. Revista Scientific, 3(9), 362-372, e-ISSN: 2542-2987. Recuperado de:

https://doi.org/10.29394/Scientific.issn.2542-2987.2018.3.9.20.362-372

Obando, M. (2019). El acompañamiento pedagógico y la praxis educativa en la educación básica en Venezuela. Revista arbitrada del CIEG Centro de Investigación y Estudios Gerenciales, (35), 94-106, e-ISSN: 2244-8330. Recuperado de:

http://www.grupocieg.org/archivos revista/Ed.35(94-106)-

Obando\%20Mar\%C3\%ADa articulo id442.pdf

Prieto, L. (1990). El Estado y la Educación en América Latina. Caracas, Venezuela: Editorial Monte Ávila. 
Nacida en Cabimas, Estado Zulia, Venezuela, el 29

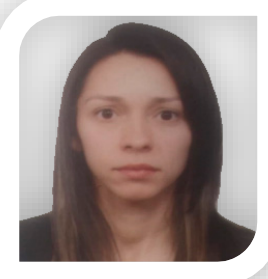
de noviembre del año 1986. Soy Ingeniero en Mantenimiento Mecánico en el año 2008; Magister Scientiarum en Docencia para la Educación Superior en el año 2017, con Componente Docente en el año 2009, logros obtenidos en la Universidad Nacional Experimental "Rafael María Baralt"; con experiencia docente desde hace más de 9 años. En este momento me desempeño como Docente a tiempo completo en la Universidad Nacional Experimental "Rafael María Baralt" (UNERMB). 


\section{Maibelyn del Carmen Acosta Vega}

e-mail: maibelyn19@gmail.com

Nacida en Cabimas, Estado Zulia, Venezuela, el 21 de

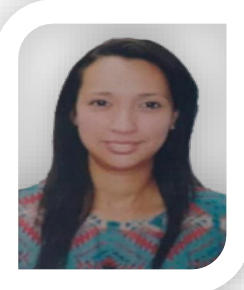

octubre del año 1984. Soy Ingeniero en Mantenimiento

Mecánico en el año 2007, Magister Scientiarum en

Docencia para la Educación Superior en el año 2017;

con Componente Docente en el año 2010, logros

obtenidos en la Universidad Nacional Experimental

"Rafael María Baralt", con experiencia docente desde hace más de 11 años.

Actualmente Docente a tiempo completo en la Universidad Nacional Experimental "Rafael María Baralt" (UNERMB). 